\title{
Measurement of Free Versus Total Therapeutic Monoclonal Antibody in Pharmacokinetic Assessment is Modulated by Affinity, Incubation Time, and Bioanalytical Platform
}

\author{
Jeffrey J. Talbot, ${ }^{1,2}$ Dominador Calamba, ${ }^{1}$ Melody Pai, ${ }^{1,3}$ Mark Ma, ${ }^{1}$ and Theingi M. Thway ${ }^{1,4}$
}

\begin{abstract}
Received 22 May 2015; accepted 15 July 2015; published online 12 August 2015
ABSTRACT. Decisions about efficacy and safety of therapeutic proteins (TP) designed to target soluble ligands are made in part by their ex vivo quantification. Ligand binding assays (LBAs) are critical tools in measuring serum TP levels in pharmacokinetic, toxicokinetic, and pharmacodynamic studies. This study evaluated the impact of reagent antibody affinities, assay incubation times, and analytical platform on free or total TP quantitation. An ELISA-based LBA that measures monoclonal anti-sclerostin antibody (TPx) was used as the model system. To determine whether the method measures free or total TPx, the effects of $K_{\text {on }}, K_{\text {off }}$, and $K_{\mathrm{D}}$ were determined. An 8:1 molar ratio of sclerostin (Scl) to TPx compared to a 1:1 molar ratio produced by rabbit polyclonal antibodies to TPx was required to achieve $\mathrm{IC}_{50}$, a measure of TPx interference effectiveness, making it unclear whether the ELISA truly measured free TPx. Kinetic analysis revealed that $\mathrm{Scl}$ had a rapid dissociation rate $\left(K_{\text {off }}\right)$ from TPx and that capture and detection antibodies had significantly higher binding affinities $\left(K_{\mathrm{D}}\right)$ to TPx. These kinetic limitations along with long ELISA incubation times lead to the higher molar ratios (8:1) required for achieving $50 \%$ inhibition of TPx. However, a microfluidic platform with the same reagent pairs required shorter incubations to achieve a lower $\mathrm{Scl} \mathrm{IC}_{50}$ molar ratio (1:1). The findings from this study provide the bioanalytical community with a deeper understanding of how reagent and platform selection for LBAs can affect what a particular method measures, either free or total TP concentrations.
\end{abstract}

KEY WORDS: affinity and kinetics; association and dissociation; free versus total; interference; ligand binding assay.

\section{INTRODUCTION}

The ligand binding assay (LBA) is a widely used tool to quantitate therapeutic proteins (TP) or soluble ligands/ protein biomarkers in biological matrixes obtained from pharmacokinetic (PK), pharmacodynamic (PD)/biomarker, or toxicokinetic (TK) studies. Therapeutic monoclonal antibodies (mAbs) are an increasingly important component of the therapeutic portfolios across the biotechnology industry, with more than $25 \mathrm{mAbs}$ already having regulatory agency approval and an additional 100 in development pipelines $(1,2)$. Because of the non-covalent interaction between TPs and their soluble ligands, multiple forms of TP and ligands can exist in vivo including free TPs (not bound to ligand), free ligands, and monovalent or bivalent TP/ligand complexes

\footnotetext{
${ }^{1}$ Department of Pharmacokinetic and Drug Metabolism, Amgen Inc., One Amgen Center Drive, Thousand Oaks, California 91320, USA.

${ }^{2}$ Present Address: Regeneron Pharmaceuticals, 81 Columbia Turnpike, Rensselaer, New York 12144, USA.

${ }^{3}$ Present Address: Department of Molecular Biology Institute, University of California at Los Angeles, Los Angeles, California, USA.

${ }^{4}$ To whom correspondence should be addressed. (e-mail: theingi.thway@amgen.com)
}

$(3,4)$. Free TPs are entities that are able to exert a biological function. Because of the bivalency of antibodies, free TP can include both unbound and partially bound forms (one ligand molecule bound to the TP) while TP assessed by total method can measure all TPs regardless of their ligand binding status (bound, partially bound, and unbound) $(3,4)$.

The decision to develop and implement methods that measure free or total TPs in matrix depends on multiple factors such as the study design, the stage of therapeutic development and limitations on relevant reagents, and the specific needs of the program. In most PK and TK studies, a free TP method is preferred and typically employed to understand target protein coverage, whereas a total TP method may be used to measure TPs in some discovery TK studies to understand the relationship between total TP concentrations and adverse effects $(5,6)$. Collectively, these data aid in developing accurate PK models for exposure determination, human dose prediction/selection, pharmacokinetic estimates, and target coverage at various drug development stages (1,7-9). Consequently, one of the greatest challenges currently presented to bioanalytical scientists is to develop a LBA method that specifically measures free TPs and does not unintentionally disturb the dynamic equilibrium of the target/TP complex. Commonly used assay formats to measure the free TP concentrations include the use of 
different clones of neutralizing anti-TP antibodies directed against idiotypic (Id) region of $\mathrm{TP}$ as capture and detection reagents (4-6). Ligand bound to TP would interfere with binding of idiotypic antibodies to TP; thus, these types of reagents are preferable in designing ELISA methods to measure free TP. Additional formats may include making use of recombinant ligands or target proteins as capture or detection reagents (10). By using neutralizing idiotypic antibodies or target proteins, there is an assumption that the method is capable of measuring free TPs $(3,5)$. However, this is not entirely accurate, and only inhibition experiments combined with kinetic data can reveal what target species a particular method measures.

During method development or pre-study method validation, interference (inhibition) tests are often conducted to determine the degree of interference in measurement of TP in the presence of the endogenous ligands, or if the method measures free versus total concentrations of the analyte of interest (11). Typically, laboratories conduct the interference tests for TP measurements in one of the following two approaches: (1) by spiking increasing concentrations of soluble proteins (often the recombinant form) targeted by TPs into two to three varying levels of spiked TP concentrations such as lower limit of quantification (LLOQ), mid-level quality control (MQC), or upper limit of quantification (ULOQ) or (2) by spiking increasing molar ratios of soluble proteins (often the recombinant form) into one concentration of spiked TP, normally at high-quality control (HQC). Determining whether the soluble proteins interfere with the accurate measurement of TP is often based on whether the positive or negative bias/ recoveries were observed with increasing soluble protein concentrations. In addition, the second approach can provide the standard measure of interference effectiveness, known as $\mathrm{IC}_{50}$, i.e., the molar ratio of target to $\mathrm{TP}$ that inhibits the detection of TP by $50 \%$. This measure can be used to characterize whether a particular ELISA method measures free TPs. For bivalent monoclonal TPs, an $\mathrm{IC}_{50}$ of $<2: 1$ molar ratio (ligand to TPs) suggests that the method truly measures free TP concentrations. However, molar ratios of >5:1 (ligand to TPs) are often observed for $\mathrm{IC}_{50}$, hence posing the question of what a particular method is measuring free or total TPs. It was hypothesized that the combination of kinetic ( $K_{\text {on }}$ or $\left.K_{\text {off }}\right)$ and affinity $\left(K_{\mathrm{D}}\right)$ differences between the TP and the ligand plays a major role in shifting of $\mathrm{IC}_{50}$. To investigate, we used a validated ELISA method that quantitates the free form of TPx, a bivalent therapeutic $\mathrm{mAb}$ candidate. TPx is a humanized anti-sclerostin antibody designed to bind and prevent it from binding and activating its cognate receptor. Sclerostin $(\mathrm{Scl})$ is a protein secreted mainly by osteocytes and a negative regulator of bone formation (12). The goal of this study was to examine the role kinetics and antibody affinities play in interference test and to definitively conclude whether this method measures free or total TPx. Additionally, how the interactions of reagent and assayed proteins affect accurate quantitation of free TPx during the PK/ PD assessment was also assessed. This study illuminates complications that most bioanalytical scientists encounter in providing method-related information to pharmacokinetic modeling scientists who further use these concentration-time data in their PK modeling efforts. This article also conveys crucial insights on how the obstacles can be tackled so that accurate and translationally relevant method-related information can be delivered to peers in the bioanalytical world, PK modelers, and clinical pharmacologists.

\section{MATERIALS AND METHODS}

\section{Reagents}

The following antibodies were generated at Amgen Inc. (Thousand Oaks, CA), in murine systems against the indicated antigen, and clonally selected to be monoclonal in nature: (A) anti-human $\mathrm{IgG} \mathrm{Fc}$, (B) a humanized anti-human scl (TPx), and (C) two neutralizing antibodies (clone 1 and clone 2) against TPx. Clone $1 \mathrm{mAb}$ was used as a capture reagent, and clone $2 \mathrm{mAb}$ labeled with horse radish peroxidase (HRP) was used as a detection antibody. The recombinant human Scl was cloned, expressed, purified, and biotinylated, internally at Amgen Inc. Streptavidin-coated bioassay 200 compact discs (CDs) were purchased from Gyros (Uppsala, Sweden). Pooled human serum was purchased from BioreclamationIVT (NY, USA). Streptavidincoated Octet biosensor tips (cat. no. 18-5021) were purchased from Pall ForteBio and kinetics assay plates (cat. no. 655209) from Greiner BioOne (Germany). All kinetic experiments were performed on the Octet Red 384 (ForteBio, cat. no. 305101 ) in assay buffer (phosphate-buffered saline (PBS), 1\% bovine serum albumin (BSA).

\section{Bioanalytical Method Procedures}

\section{Validated Ligand Binding Assay to Measure Anti-human Sclerostin Antibody X}

The ELISA method to measure TPx had been previously reported (13). Briefly, microplates (96 wells) were coated with $2 \mu \mathrm{g} / \mathrm{ml}$ of clone $1 \mathrm{mAb}$ (Lot No. 69890-012) in sodium bicarbonate buffer. Standard calibrators (STD) and quality controls (QC) were prepared by spiking stock anti-human sclerostin antibody (TPx, Lot No. A078089) in 100\% pooled human serum using Tecan liquid handling robot (Tecan Systems, San Jose, CA). The STD, QC, and blank were diluted 1:50 in assay buffer (1X PBS with $0.5 \mathrm{M} \mathrm{NaCl}$ and $0.5 \%$ Tween 20 ) prior to addition into the coated 96-well microtiter plate and incubated for $1.8 \mathrm{~h} \pm 10 \mathrm{~min}$. After a wash step, $100 \mu \mathrm{l}$ of $5 \mathrm{ng} / \mathrm{ml}$ HRP-conjugated neutralizing murine antibody against TPx (clone 2, Lot No. 69890-005 or 69890006) was added to the wells and incubated at $25^{\circ} \mathrm{C}$ for $1.3 \mathrm{~h} \pm 10 \mathrm{~min}$. The TMB peroxide HRP substrate solution (Kirkegaard and Perry Laboratories Inc., MD) was added after the final wash step and incubated for $16 \pm 1 \mathrm{~min}$. The color development was stopped by acidification, and the optical density (OD) was measured at $450 \mathrm{~nm}$ with reference to $650 \mathrm{~nm}$ using Molecular Devices Spectramax 340PC microplate reader equipped with SOFTmax Pro version 4.3 or higher software. The assay's dynamic range is from 50 to $2495 \mathrm{ng} / \mathrm{ml}$. The concentration-instrument response data was fitted using a four-parameter logistic (Auto Estimate) regression model without a weighting factor using Watson ${ }^{\text {TM }}$ LIMS v 7.0.0.01 or higher. 
Validated Immunoassay to Measure Total Sclerostin

A chemiluminescence ELISA method was used to measure Scl. Briefly, microplates (96 wells) were coated with a humanized anti-Scl antibody (clone A, Lot No. 021207GD, Amgen, Inc. CA), a different clone from TPx. STDs and QCs were prepared by spiking recombinant human Scl (Lot No. 14062706, Amgen Inc., CA) in I-Block buffer with $100 \mu \mathrm{g} / \mathrm{ml}$ mouse IgG. The STDs, QCs, and blank were pre-treated 1:4 in assay buffer (I-Block) followed by treatment with an alkaline solution and then neutralized by a sodium phosphate buffer before being added to the coated microplate. After a wash step, HRP-conjugated humanized anti-Scl antibody (clone Z, Lot No. HRPAMG685030907HSM, Amgen Inc., CA) which was different from TPx was added to the wells for detection of the captured Scl. After a final wash step, the SuperSignal $^{\circledR}$ ELISA FEMTO substrate solution (Pierce, IL) was added to the wells. The substrate in the presence of HRP produced a chemiluminescence signal that was proportional to the amount of $\mathrm{Scl}$ bound to the capture reagent. The instrument response (RLU) was measured using Molecular Devices Spectramax 340PC microplate reader equipped with SOFTmax Pro Version 4.3 or higher software. The assay's dynamic range is from 0.3 to $75 \mathrm{ng} / \mathrm{ml}$.

\section{Microfluidic Platform to Measure TPX}

A MFP procedure similar to previously described method in Magana et al. (14) was used to measure TPx in MFP with the following exceptions: the STD and QC were made by spiking TPx into I-Block buffer and diluted 1:10 in assay buffer ( $1 \mathrm{X}$ PBS with $1 \mathrm{M} \mathrm{NaCl}, 0.5 \%$ Tween 20 , and $1 \%$ BSA) prior to loading into a Bioaffy $200 \mathrm{CD}$. Biotinylated murine monoclonal antibody against TPx (clone 1, Lot No. P49622.5) was used as the capture reagent, and Alexa Fluor-labeled murine monoclonal against TPx (clone 2, Lot No. P49662.5) was used as the detection antibody. Both mAbs are neutralizing antibodies against TPx and same clones as immunoassay to measure TPx. Biotinylated clone 1 was diluted in assay buffer (1X PBS with $1 \mathrm{M} \mathrm{NaCl}, 0.5 \%$ Tween 20 , and $1 \% \mathrm{BSA}$ ) to $5 \mu \mathrm{g} / \mathrm{ml}$, and Alexa Fluor-labeled clone 2 centrifuged at approximately 14,000 RPM for $4 \mathrm{~min}$ and diluted in assay buffer to approximately $3 \mu \mathrm{g} / \mathrm{ml}$. The "Bioaffy 2003 step vp4 1\%PMT wiz" template was used to design the assay as in Thway et al. (6). Experiments were done using GyrosLab xP workstation equipped with GyrosLab Control version 5.2.0 (Gyros ${ }^{\circledR}$, Uppsala, Sweden). The assay's dynamic range is from 50 to $40,000 \mathrm{ng} / \mathrm{ml}$. The concentration-instrument response data was fitted using a five-parameter logistic (Auto Estimate) regression model with a weighting factor of $1 / \mathrm{Y}$ using Watson ${ }^{\text {TM }}$ LIMS v 7.4.

\section{Interference Experiments Using Spiked Serum Samples}

The immunoassay to measure TPx included STDs and QCs which were prepared in 100\% human serum. A TPx spiked serum sample was prepared at $1198 \mathrm{ng} / \mathrm{ml}, 2 \mathrm{X}$ the final concentration. Similarly, interfering molecules (Scl or polyclonal anti-TPx antibodies (pAbs)) were prepared at increasing molar ratios to TPx in $2 \mathrm{X}$ final concentration solutions.
The molar ratios (interfering molecule to TPx, respectively) of pAbs ranged from 0.02 to 20 , and those of $\mathrm{Scl}$ ranged from 0.001 to 10 . After spiked sample preparation, an equal volume of $2 \mathrm{X}$ TPx was mixed with either $2 \mathrm{X} \mathrm{Scl}$ or $2 \mathrm{x}$ antiTPx pAbs to create a final $1 \mathrm{x}$ concentration. The final TPx concentration was $599 \mathrm{ng} / \mathrm{ml}$ and fell in the middle of the assay range. The samples were mixed and incubated at $37 \pm 5^{\circ} \mathrm{C}$ for $30 \mathrm{~min}$ to $1 \mathrm{~h}$ and frozen at $-70^{\circ} \mathrm{C}$ until analysis. Percent interference was determined by comparing percent recovery of TPx samples containing each interfering molecule to the negative control sample (only TPx) that did not contain an interfering molecule. For the microfluidic platform immunoassay, the molar ratio of TPx to Scl ranged from 0.03 to 30 .

\section{Kinetic Experiments: Determination of Association and Dissociation Constants}

The streptavidin (SA)-coated biosensor tips were equilibrated in $1 \mathrm{X}$ PBS with $1 \%$ BSA for at least 10 min prior to starting the experiment. Experiment steps include baseline 1, loading 1 , loading 2 , baseline 2 , association, and dissociation. During baseline 1 (60 s), all of the SA biosensor tips were equilibrated in $1 \mathrm{X}$ PBS with $1 \%$ BSA. Loading 2 allowed biotinylated-anti-Fc mAb diluted at $5 \mu \mathrm{g} / \mathrm{ml}$ in $1 \mathrm{X}$ PBS with $1 \%$ BSA to bind the SA biosensor tips (200 s). During loading 2 step, all biosensor tips were transferred to wells containing $2.5 \mu \mathrm{g} / \mathrm{ml}$ TPx in 1X PBS with $1 \%$ BSA (250 s). During baseline $2(60 \mathrm{~s})$, biosensor tips were washed in assay buffer to remove unbound molecules. Association step (350 s for capture and detection, and $400 \mathrm{~s}$ for $\mathrm{Scl}$ ) placed the tips into wells containing capture, or detection, or Scl in $1 \mathrm{X}$ PBS with $1 \%$ BSA. Finally, during dissociation steps, all tips were transferred to wells containing only $1 \mathrm{X}$ PBS with $1 \%$ BSA (400 s capture and detection, $600 \mathrm{~s}$ for Scl). To generate $K_{\text {on }}$, $K_{\text {off }}$, and $K_{\mathrm{D}}$ values, six concentrations $(0.625,1.25,2.5,5,10$, $20 \mu \mathrm{g} / \mathrm{ml}$ ) of each analyte (capture, detection, or Scl) were run in parallel for each experiment; after subtracting background, the global fit for association and dissociation for all six concentrations was used to determine kinetic constant values.

The dissociation constant $\left(K_{\mathrm{D}}\right)$ is an equilibrium constant expressed in units of concentration (molarity), describing the affinity (tightness) with which the tested ligand binds to its target protein. In this case $\mathrm{Scl}$, capture, and detection Abs were compared for their affinity to TPx. $K_{\mathrm{D}}$ is the equilibrium concentration at which half of the ligands in solution have formed a complex with their target protein. A lower $K_{\mathrm{D}}$ indicates greater affinity.

$K_{\text {on }}$ and $K_{\text {off }}$ are measured in units of time $\left(\mathrm{M}^{-1} \cdot \mathrm{min}^{-1}\right.$, or $\min ^{-1}$, respectively) and describe the rate at which ligands bind to form a complex $\left(K_{\mathrm{on}}\right)$ with their target protein, or dissociation of the ligand/target protein complex $\left(K_{\text {off }}\right)$. For the scope of this work, the relationships can be described as $K_{\mathrm{D}}=K_{\text {off }} / K_{\text {on }}$.

\section{Kinetic Competition Experiments}

The streptavidin biosensor tips were equilibrated in $1 \mathrm{X}$ PBS with $1 \%$ BSA for at least $10 \mathrm{~min}$ prior to starting the experiment. The kinetic competition experiment had four steps: baseline, loading, association, and dissociation. All 
streptavidin biosensor tips were equilibrated in $1 \mathrm{X}$ PBS with $1 \%$ BSA during baseline step (60 s). Biotinylated Scl diluted to $2.5 \mu \mathrm{g} / \mathrm{ml}$ in assay buffer was bound to streptavidin biosensor tips during loading (300 s). Association step allows binding of TPx at $2.5 \mu \mathrm{g} / \mathrm{ml}$ in $1 \mathrm{X}$ PBS with $1 \%$ BSA to biotinylated $\mathrm{Scl}(400 \mathrm{~s})$. During association, the $0 \%$ bound TPx tips were transferred to wells containing only $1 \mathrm{X}$ PBS with $1 \%$ BSA, without TPx. Finally, competition occurred during dissociation step (3600 s). During dissociation competition, tips were transferred to wells containing neutralizing anti-Id capture antibody at 1 or $20 \mu \mathrm{g} / \mathrm{ml}$ in assay buffer. During dissociation, the $0 \%$ bound TPx (without TPx) and the $100 \%$ bound TPx $(2.5 \mu \mathrm{g} / \mathrm{ml}$ TPx $)$ tips were transferred to wells containing only assay buffer, but no competing capture antibody.

\section{RESULTS}

The Molar Ratio of Recombinant Sclerostin or Polyclonal Antibodies Varies Depending on Nature of Interfering Protein

During the pre-study method validation, interference experiments were run using polyclonal rabbit antibodies (pAbs) raised against the complementarity determining region (CDR) of TPx to determine if the accurate measurement of TPx can be affected by the presence of anti-drug antibodies in a study sample. The pAbs were raised in rabbit against the Fab portion of TPx and used as a positive control for immunogenicity testing; it has previously been established that they can neutralize TPx. Increasing the molar ratio of pAbs to TPx decreased the quantification of TPx in ELISA (pAb in Fig. 1) indicating that the ELISA measures free TPx (Fig. 2 first panel). The $\mathrm{IC}_{50}$ of the pAbs corresponded with a 1:1 molar ratio of pAbs to TPx (Fig. 1).

With respect to endogenous ligand interference, it was anticipated that occupancy of either arm of TPx with the ligand would interfere with binding of both capture and/or detection antibodies and prevent assay signal based on the method format (Fig. 2 middle panel). Therefore, in addition to pAbs, an interference experiment was also run with increasing amounts of human recombinant Scl. Approximately 8 molar ratio of $\mathrm{Scl}$ was required for $50 \%$ inhibition of the TPx (Fig. 1). The 1:1 molar ratio of pAb:TPx aligned with the theoretical perspective: that the ELISA can only measure TPx that is not bound to neutralizing anti-drug antibody (ADA) (i.e., ADA against CDR of TPx). However, the 8:1 $\mathrm{IC}_{50}$ molar ratio of Scl:TPx was unanticipated. As there was a discrepancy in observation for the two interfering molecules, the ability of this ELISA method to measure free TPx was uncertain. To further characterize this method, the kinetic analyses were performed.

\section{Kinetic and Affinity Comparison of Reagents Used in LBA}

As first described by Guldberg and Waage in 1864, most biological binding interactions are reversible, and even molecules which bind each other avidly have some portion of unbound molecules in solution (15). Therapeutic antibodies are typically designed to bind their targets very strongly, with typical dissociation constants $\left(K_{\mathrm{D}}\right)$ ranging from low

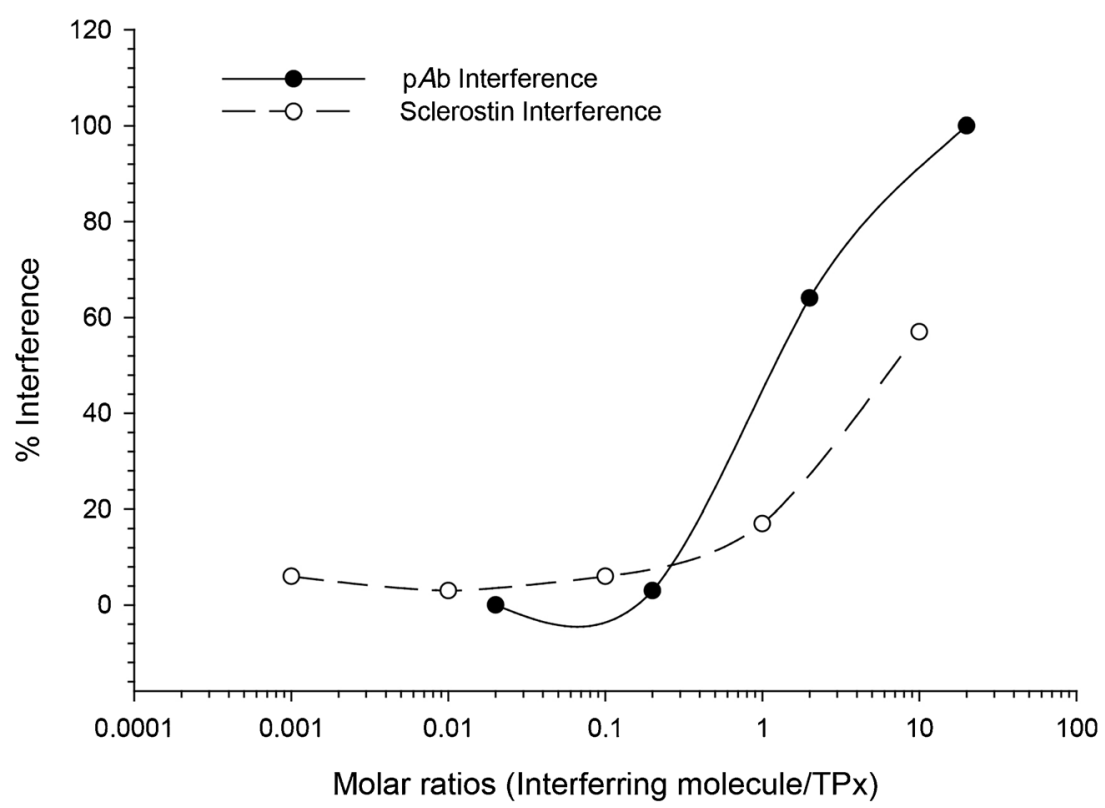

Fig. 1. Percent interference-molar ratio profile of anti-sclerostin antibody (TPx) in the presence of either polyclonal antibodies ( $\mathrm{pAb}$ ) against Fab region of TPx (filled circles) or recombinant sclerostin (open circles) as measured by microplate-based ELISA designed to detect free levels of TPx. Percent interference represents the percentage of decrease in detected TPx in the presence of interfering proteins of either $\mathrm{pAb}$ or sclerostin. Both $\mathrm{pAb}$ and sclerostin had a concentration-dependent ability to interfere with detection of free TPx. At similar molar ratios, $\mathrm{pAb}$ had a greater ability to interfere with free TPx detection. $\mathrm{IC}_{50}$ was determined by the molar ratio of interfering protein that resulted in a $50 \%$ decrease in detection of free TPx. The $\mathrm{IC}_{50}$ for $\mathrm{pAb}$ and sclerostin are 1:1 and 8:1, respectively 


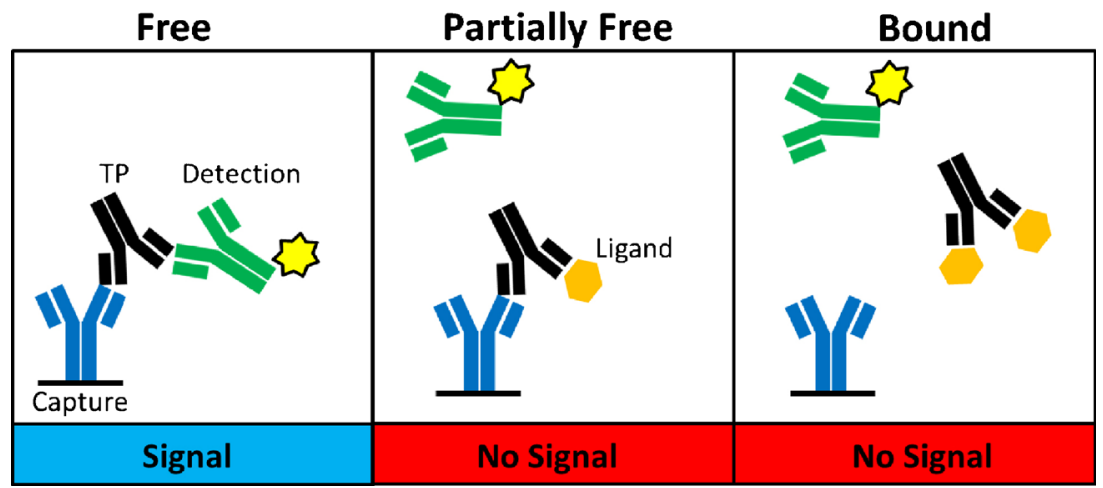

Fig. 2. Diagram describing the three possible species of mAbs (free, 1 arm bound, both arms bound) which may be present in study samples and the ability of the free TP method to quantitate each form. Two different clones of neutralizing anti-idiotypic monoclonal antibodies were used as capture and detection reagents. The ligand binding site of ligand to TP overlaps with that of the capture and detection antibodies, making TP undetectable if a ligand is bound to one or both arms of TP (partially bound and totally bound, respectively)

nanomolar to picomolar levels (3). To better understand why 8:1 molar ratio was necessary for $50 \%$ inhibition, association $\left(K_{\mathrm{on}}\right)$, dissociation $\left(K_{\mathrm{off}}\right)$, and the dissociation constant $\left(K_{\mathrm{D}}\right)$ between Scl and TPx were determined and compared to those of capture/detection and TPx. $K_{\mathrm{D}}$ describes the affinity of TPx's binding partners (Scl, capture and detection Abs) for TPx, whereas $K_{\text {on }}$ and $K_{\text {off }}$ describe the rate at which Scl or capture and detection Abs bind and form a complex with TPx $\left(K_{\text {on }}\right)$, or the rate at which this complex dissociates $\left(K_{\text {off }}\right)$.

Looking at the rates of complex formation, both capture and detection antibodies (6.01E+05 1/Ms each) associate and form a complex with TPx more rapidly when compared to the rate at which Scl forms a complex with TPx (6.03E+04 1/Ms) (Table I). Additionally, once complexed, the capture or detection antibodies dissociated $\left(K_{\mathrm{off}}\right)$ from TPx much more slowly $(<1.0 \mathrm{E}-071 / \mathrm{s}$ and $1.75 \mathrm{E}-071 / \mathrm{s}$, capture and detection, respectively) than the complex formed by Scl and TPx (6.22E -07 1/s) (Table I and Fig. 3). Most interestingly, looking at affinity, the complexes formed by TPx and the capture and detection antibodies were much stronger $(<1.0 \mathrm{pM}$ and $<1.0$ $\mathrm{pM}$, respectively) than those formed by TPx and Scl (7.04 pM) (Table I).

\section{Dynamic Equilibrium of the Sclerostin/TPx Complex is Altered by Capture and Detection Antibodies}

Having determined that the reagent antibodies/TPx complex is of greater affinity than the TPx/Scl complex (Table I), we examined the possibility that the capture antibody could out-compete Scl from the TPx/Scl complex in ELISA format. On the biosensor tip, immobilized Scl was bound by TPx, and the complex was introduced to one of two different concentrations of capture antibody ( 1 and $20 \mu \mathrm{g} / \mathrm{ml})$ to compete with Scl (Fig. 4, conditions 3 and 4) and compared to without the addition of capture reagent (condition 1, 100\% TPx bound to $\mathrm{Scl}$ ) as well as without TPx and capture reagent (condition 4, a negative control). In this experimental setup, when the capture antibody successfully competes TPx away from Scl, there is a loss of complex (thickness/mass) on the biosensor tip resulting in less optical flux and a decreased value on the $Y$-axis (Fig. 4). Capture antibody at $1 \mu \mathrm{g} / \mathrm{ml}$ rapidly displaces TPx in complexes with Scl. Increasing the concentration of capture antibody (up to $20 \mu \mathrm{g} / \mathrm{ml}$ ) further alters the newly established dynamic equilibrium and additionally accelerates this process (Fig. 4 condition 3). To determine relative change, a positive control without any competing capture antibody (Fig. 4 condition 1) and a negative control without any TPx or capture antibody (Fig. 4 condition 4) were run to indicate relative competitiveness of the capture antibody.

\section{Short Sample Incubation Time Reduces the Impact of Kinetic Variables and Changes $\mathrm{IC}_{50}$ Ratio}

We tested the ability of Scl to interfere in measurement of TPx using the MFP to examine the impact of assay incubation time and dilution. Unlike the plate-based ELISA which has typical sample incubation time of $60 \mathrm{~min}$ each with capture and then detection antibody (Fig. 1), the MFP is a

Table I. Binding Characteristics of Capture, Detection Antibody, and Sclerostin to Anti-sclerostin Antibody (TPx)

\begin{tabular}{|c|c|c|c|c|c|c|}
\hline Biotinylated reagent & Second immobilized reagent & Analyte & $K_{\text {on }}(1 / \mathrm{Ms})$ & $K_{\text {off }}(1 / \mathrm{s})$ & $K_{\mathrm{D}}(\mathrm{M})$ & $K_{\mathrm{D}}(\mathrm{pM})$ \\
\hline Anti-human IgG Fc & TPx & Anti-Id (capture $\mathrm{Ab}$, clone $1^{a}$ ) & $6.01 \mathrm{E}+05$ & $<1.0 \mathrm{E}-07$ & $<1.0 \mathrm{E}-12$ & $<1.0$ \\
\hline Anti-human IgG Fc & $\mathrm{TPx}$ & Anti-Id (detection $\mathrm{Ab}$, clone $2^{a}$ ) & $6.01 \mathrm{E}+05$ & $1.75 \mathrm{E}-07$ & $<1.0 \mathrm{E}-12$ & $<1.0$ \\
\hline Anti-human IgG Fc & TPx & $\mathrm{rHu} \mathrm{Scl}$ & $6.03 \mathrm{E}+04$ & $6.22 \mathrm{E}-07$ & $7.04 \mathrm{E}-12$ & 7.04 \\
\hline
\end{tabular}

Parameters examined included association and dissociation kinetics $\left(K_{\mathrm{on}}\right.$ and $\left.K_{\text {off }}\right)$ as well as binding affinity $\left(K_{\mathrm{D}}\right)$ of capture and detection antibodies and sclerostin to TPx. Affinity for TPx is significantly different between the antibody reagents and sclerostin

${ }^{a}$ Neutralizing $\mathrm{mAb} ; \mathrm{rHu} \mathrm{Scl}$ : recombinant human sclerostin 

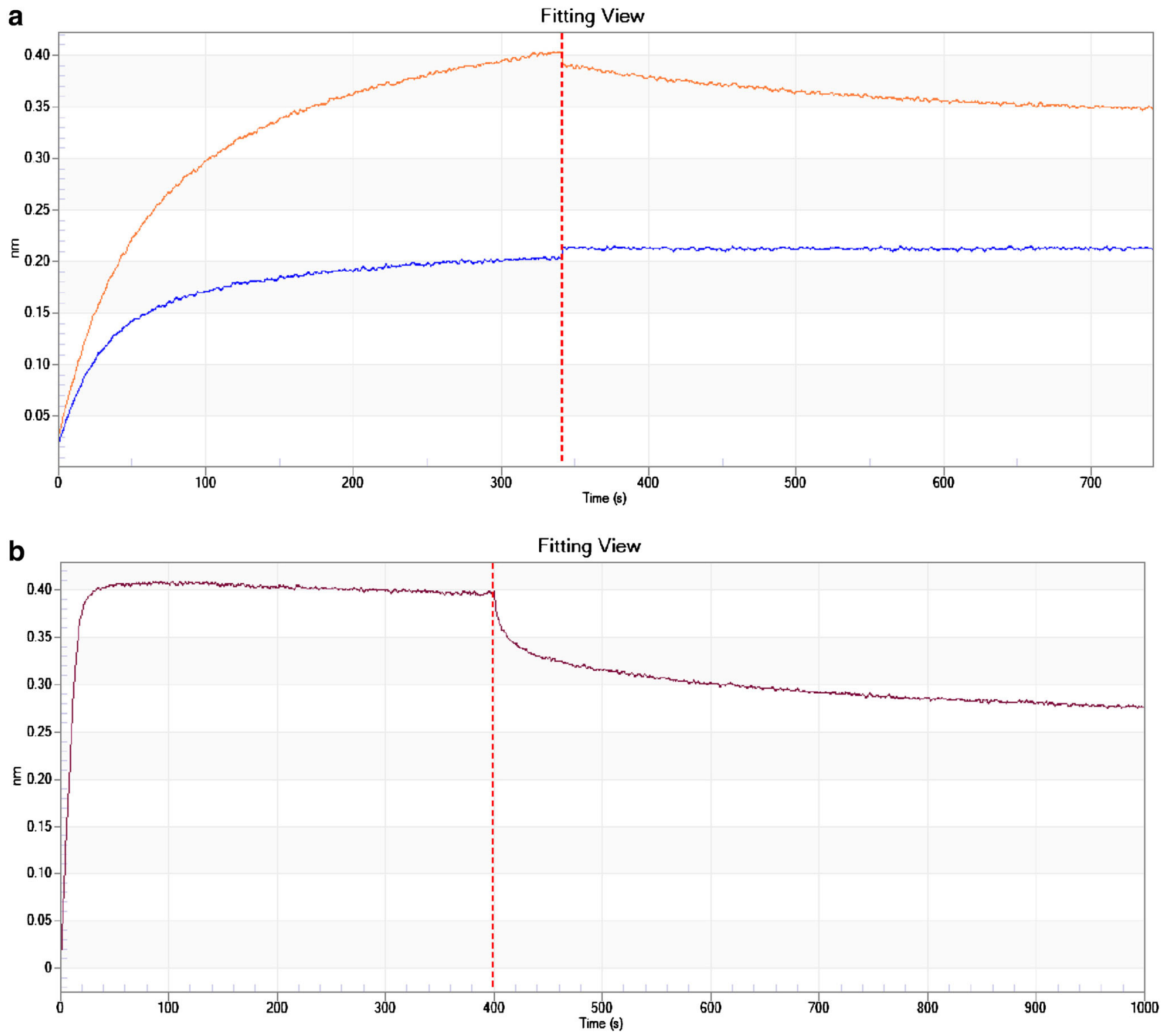

Fig. 3. Real-time plots of antibody reagents (a blue line, capture; orange line, detection) and sclerostin (b burgundy line) association and dissociation from antisclerostin antibody (TPx). Capture, detection, and sclerostin were used at $2.5 \mu \mathrm{g} / \mathrm{ml}$ resulting in a saturation of TPx with sclerostin (b) and sub-saturation levels of TPx by capture and detection (a). The more rapid dissociation of sclerostin (b) further illustrates the different affinities of the reagent antibodies and sclerostin to TPx

\begin{tabular}{|cccc|}
\hline Condition & $\begin{array}{c}\text { Sclerostin } \\
(2.5 \mu \mathrm{g} / \mathrm{ml})\end{array}$ & $\begin{array}{c}\text { TPx } \\
(2.5 \mu \mathrm{g} / \mathrm{ml})\end{array}$ & $\begin{array}{c}\text { Anti-ld } \\
\text { capture }\end{array}$ \\
\hline 1 & Yes & Yes & No \\
2 & Yes & Yes & $1 \mu \mathrm{g} / \mathrm{ml}$ \\
3 & Yes & Yes & $20 \mu \mathrm{g} / \mathrm{ml}$ \\
4 & Yes & No & No \\
\hline
\end{tabular}

column-based flow-through assay, and samples flow across capture antibody column for only $6 \mathrm{~s}$ prior to detection antibody addition. There was a significant shift in the $\mathrm{IC}_{50}$ for $\mathrm{Scl}$ in the MFP-based method versus the plate-based method.
In the MFP, the $\mathrm{IC}_{50}$ ratio for $\mathrm{Scl}$ to $\mathrm{TPx}$ was $1: 1$. About eightfold less, Scl was necessary to inhibit the detection of $50 \%$ of TPx in the MFP method (Fig. 5) compared to the ELISA method (Fig. 1). This data indicated that in the 


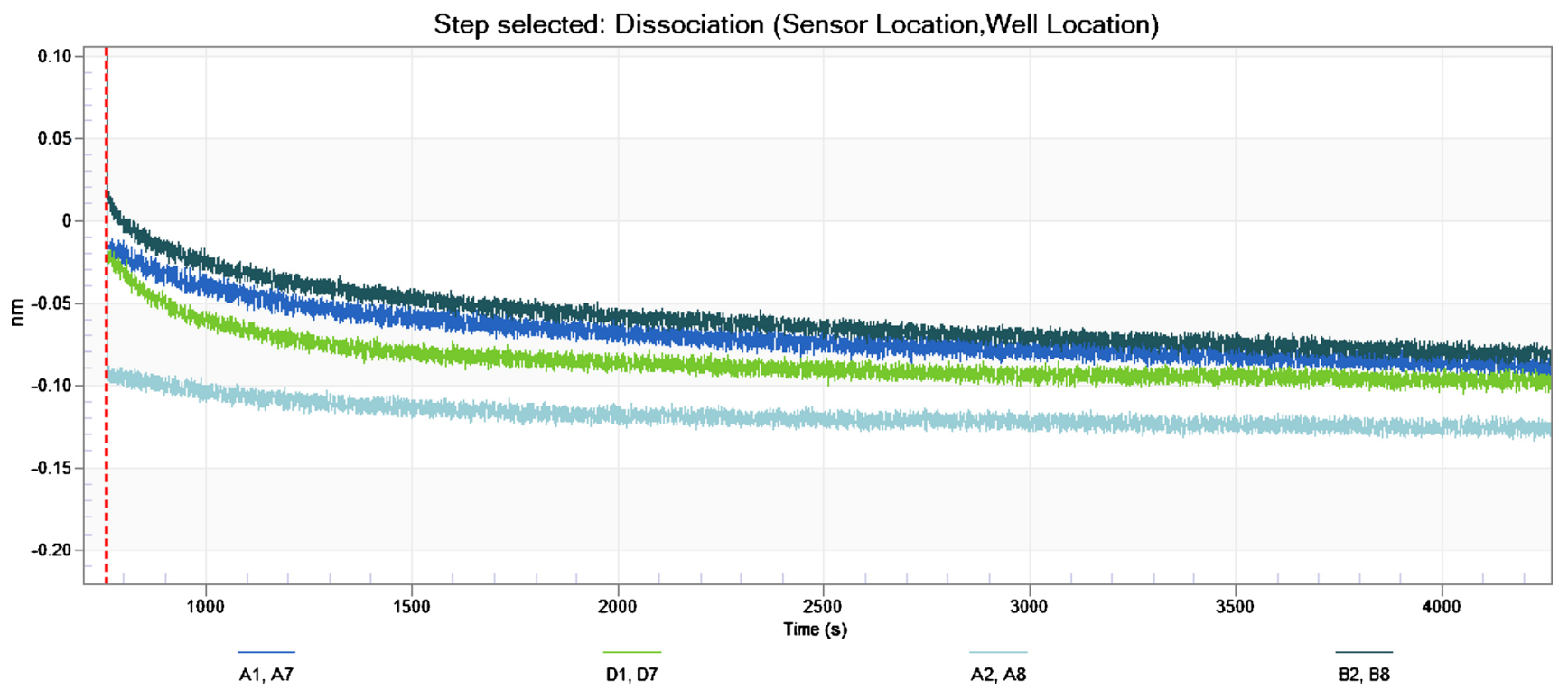

Fig. 4. Real-time plot of capture antibody and sclerostin competing for anti-sclerostin antibody (TPx) binding sites. Sclerostin was immobilized onto streptavidin biosensor tips, and TPx was allowed to bind in conditions 1-3. In competitive assay conditions (2 and 3), capture antibody was introduced and allowed to compete for TPx binding sites. A downward shift of the trace on the $Y$-axis represents successful competition of TPx away from sclerostin by the capture antibody. Conditions 2 and 3 include increasing amount of anti-Id capture antibody ( 1 and $20 \mu \mathrm{g} / \mathrm{ml}$, respectively) and represent increasingly competitive environments for TPx to maintain binding to sclerostin. Condition 1 does not contain capture antibody, representing a non-competitive environment with $100 \%$ TPx bound to sclerostin while condition 4 has no TPx and no capture antibody, representing complete competition, and $0 \%$ TPx bound to sclerostin

ELISA, the sustained exposure of the Scl/TPx complex to capture and detection antibodies promoted disruption of the $\mathrm{Scl} / \mathrm{TPx}$ complex, in favor of capture/TPx or detection/TPx complexes. This result suggested that the sample incubation time in MFP platform was shortened enough that Scl/TPx complexes remain as a complex and capture reagent did not have sufficient time to bind to TPx once Scl dissociates from complexes.

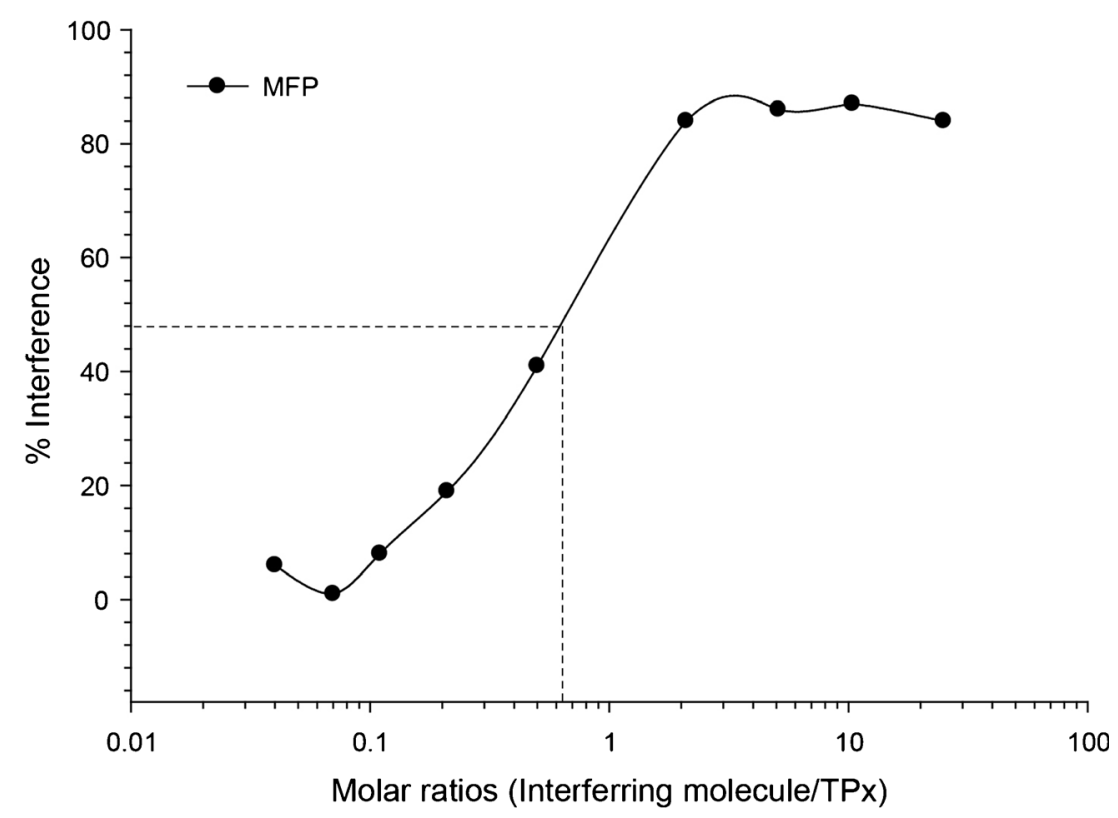

Fig. 5. Interference assay data generated using the microfluidic platform $(M F P)$. The molar ratio of the $\mathrm{IC}_{50}$ from interference data generated using the MFP and the same reagents as the plate-based ELISA was 1:1, sclerostin to anti-sclerostin antibody (TPx), respectively. Percent interference represents the percentage of decrease in detected TPx in the presence of increasing molar ratio of sclerostin. This differed significantly from the plate-based ELISA interference data which yielded 8:1, sclerostin to TPx, respectively. At low ratios $(0.1: 1)$, sclerostin has no ability to interfere with TPx, and at ratios equal to or exceeding 2:1, TPx signal is interfered by approximately $90 \%$ 


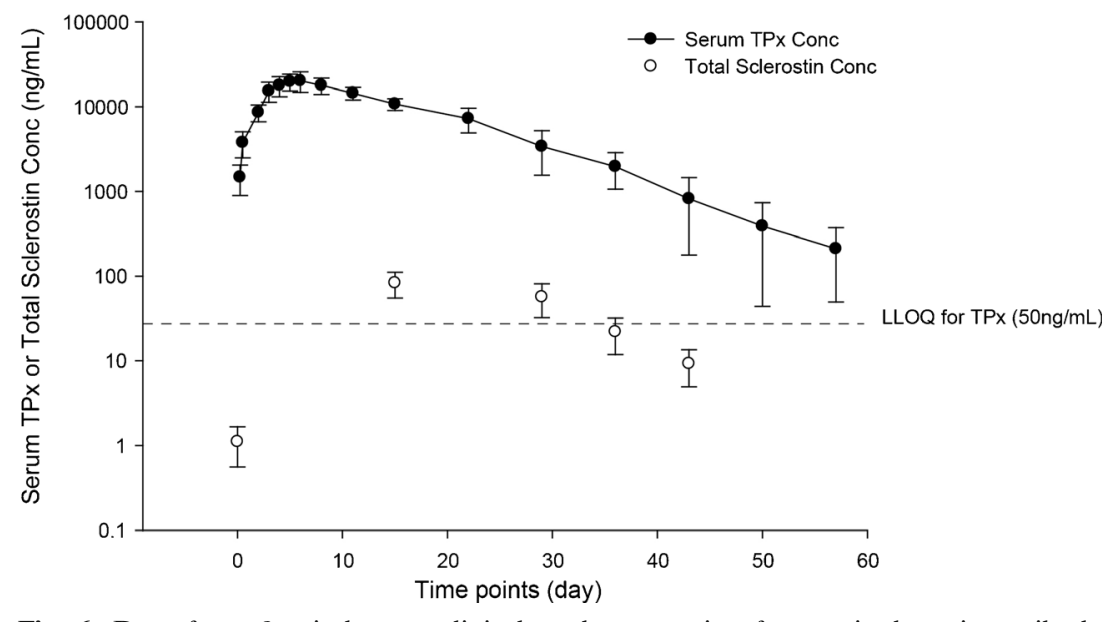

Fig. 6. Data from first-in-human clinical study measuring free anti-sclerostin antibody $(T P x)$ and total sclerostin using colorimetric ELISA (for TPx) and planar chemiluminescence-based ELISA (for sclerostin). Free TPx levels peak at approximately study day 8 and decrease at a constant rate through study day 60. A dose-induced sclerostin accumulation peaks at study day 15 and decreases at approximately the same rate as TPx for the remaining time points. After the dosing of TPx, sclerostin levels are elevated above the untreated baseline (day 0 ). The ratio of sclerostin to TPx is always very low indicating that in vivo TPx is predominantly free

\section{Most TPx is Free or Partially Bound by Sclerostin In Vivo}

The 8:1 interference-molar ratio generated using $\mathrm{Scl}$ as the interfering protein indicated that the ELISA may overestimate free TPx concentration. Data from a clinical study in which the serum TPx and total Scl concentration was measured were overlaid to determine if there may have been an overestimation of free TPx in samples. In human clinical samples, dosing with TPx produced a correlated lagging spike in serum Scl levels (Fig. 6). However, throughout the course of the study, serum TPx levels persist at significantly higher levels than Scl indicating low TPx occupancy by Scl. The mean molar ratio of Scl to TPx at day 15 was 0.05:1. Even at maximum TPx occupancy on day 57, the mean molar ratio of Scl to TPx was $0.27: 1$. This result indicated that TPx quantification of serum samples was not being significantly impacted by elevated serum levels of $\mathrm{Scl}$ which remained fairly low.

\section{DISCUSSION}

Soluble targets such as cytokines and growth factors found circulating in the bloodstream have become common targets for therapeutic mAbs (1,2). One of the complications of targeting such proteins is the observed phenomena of target accumulation in response to drug administration. Ranging from low picograms up to micrograms per milliliter, target protein accumulation can be highly variable depending on disease and disease-associated target (16). This accumulation may be due to indirect and related effects of dosing with the therapeutic mAbs. Alternatively, FcRn-mediated recycling of the therapeutic $\mathrm{mAb}$ also effectively recycles the $\mathrm{mAb}$ /target complex, thereby promoting accumulation of the target and directly influencing serum target levels (17). While methods measuring total TPs are not impacted by fluctuations in circulating target levels, methods designed to measure free TP concentrations can be heavily influenced by dosing-mediated changes in target levels. Using the TPx ELISA designed to quantitate free TPx, the impact of affinity, sample incubation time, and platform choice were evaluated to understand how they influence accurate quantitation of free serum TPx. The ELISA was designed with the intent to measure free TPx using neutralizing anti-idiotypic antibodies as capture and detection. During interference testing in a pre-study method validation stage, differences were discovered in the molar ratios between TPx and pAbs or Scl and their ability to yield similar $\mathrm{IC}_{50}$ values. The approximately eightfold higher molar ratio of $\mathrm{Scl}$ over $\mathrm{pAbs}$ necessary to reach $\mathrm{IC}_{50}$ was not immediately explainable given that both interfering proteins should bind one or both arms of TPx and contribute to interference in the same way, based on the anti-idiotype antibodies as capture and detector.

Following up on this observation, the binding affinity of Scl to TPx was found to be lower than that of the capture and detection reagents. Additionally, the kinetic parameters, $K_{\text {on }}$ and $K_{\text {off }}$, indicated that complexes between TPx and Scl were slower to form and faster to dissociate than those between TPx and the capture or detection antibodies. Taken together, these kinetic and affinity data indicate that during the extended incubation time of the plate-based ELISA, Scl readily dissociates from TPx allowing it to form a complex with the capture antibody. This interaction between capture antibody and TPx is stronger and longer lived, leading to the higher than expected $\mathrm{IC}_{50}$ molar ratio for Scl.

To explore this hypothesis, an inhibition assay was developed using TPx bound to streptavidin biosensor tipimmobilized Scl, in which the capture antibody was introduced and allowed to compete with $\mathrm{Scl}$ for TPx/Scl complexes. Unlike the plate-based ELISA which provided a data snapshot representing the final results of the assay, the biosensor competition assay showed real-time streaming data. 
The competition assay confirmed that the capture antibody was able to effectively out-compete Scl for TPx binding sites. Indeed, the capture antibody out-competed Scl for TPx binding sites relatively soon after introduction to the TPx/ Scl complex and the new complex remained constant over time in a dose-dependent fashion.

Based on these results, it was clear that the combination of reagent affinity and their kinetics produced the unexpected 8:1 molar ratio for $\mathrm{IC}_{50}$. The interference experiment in the MFP platform reversed the molar ratio to empirically theoretical 1:1 molar ratio. The MFP was chosen since the sample incubation time between the samples and capture reagent was reduced to $6 \mathrm{~s}$ in MFP as opposed to greater than 1-h incubation periods with the capture and detection antibodies in typical ELISA. Collectively, these results suggested that the combination of reagent affinity, their kinetics, sample incubations, and method platform determine the ability of a method to measure free or total TPs.

Clinical data from a human PK study that measured the levels of free TPx and Scl found that the majority of TPx molecules remained free for most of the study, even after a dosing-induced spike and accumulation of Scl was observed. At later time points, where less TPx remained in circulation, the ratio of Scl to TPx increased, but never significantly, and never enough to bind more than approximately $25 \%$ of measured serum TPx. Regardless of whether bioanalytical methods quantitate free or total protein therapeutics, reporting accurate PK data depends on a method with high specificity. While the TPx method was designed to be a free method, experiments investigating the discrepancies found during the interference testing between the $\mathrm{pAb}$ and $\mathrm{Scl}$ indicated that the method may indeed measure free TPx with respect to high affinity ADA antibodies and possible total TPx with respect to Scl. Since the molar ratio of TPx to Scl was so high in vivo, the serum TPx concentration represents mostly free TPs.

\section{CONCLUSION}

Accurate measurement of TP is critical to the interpretation of PK/PD data in order to evaluate the relationship between TP exposure, dosing, and efficacy. Developing LBAs to measure free and total target provide bioanalytical scientists and pharmacokineticists with a more complete picture of the in vivo landscape. A few significant findings emerged from this study. First, the use of neutralizing anti-Id antibodies, recombinant ligands, or target proteins as capture and detection reagents in LBA would not automatically provide a method capable of measuring free TPs. The inhibition tests with increasing molar ratio of ligand to TP can reveal what a particular method measures. Second, a particular method may measure total TPs with respect to ligand but it can still measure free TPs with respect to ADA (i.e., TPs that are not bound to anti-drug antibody at the complementary determining region of TP). Thus, an extra level of awareness during method design is especially necessary for development of free TP methods. Without quantitative kinetic and affinity data between TP, target, and reagent antibodies, interference test can generate misleading data about what the method actually measures. As such, during the method development process, interference testing along with kinetic assessment is an important step in developing an accurate free TP method. Interestingly, in order to sustain their desired therapeutic effect, most $\mathrm{mAb}$ TPs are maintained at higher levels than their target in vivo, and so, most TP is generally free. Taken as a whole, the data from this study showed valuable information regarding method development and how to characterize what a particular method measures with respect to free and total TPs in conjunction with critical analysis of PK/PD data sets.

\section{ACKNOWLEDGMENTS}

We would like to thank Drs. Timothy Heath and Vibha Jawa (Amgen Inc., Thousand Oaks, CA) for their critical review. Financial support for this article was provided by Amgen Inc.

\section{REFERENCES}

1. Wang W, Wang EQ, Balthasar JP. Monoclonal antibody pharmacokinetics and pharmacodynamics. Clin Pharmacol Ther. 2008;84(5):548-58.

2. Dostalek $\mathrm{M}$ et al. Pharmacokinetics, pharmacodynamics and physiologically-based pharmacokinetic modelling of monoclonal antibodies. Clin Pharmacokinet. 2013;52(2):83-124.

3. Lee JW, Kelley M, King LE, Yang J, Salimi-Moosavi H, Tang MT, et al. Bioanalytical approaches to quantify "total" and "free" therapeutic antibodies and their targets: technical challenges and PK/PD applications over the course of drug development. AAPS J. 2011;13(1):99-110.

4. Kuang B, King L, Wang HF. Therapeutic monoclonal antibody concentration monitoring: free or total? Bioanalysis. 2010;2(6):1125-40.

5. Ahene AB. Application and interpretation of free and total drug measurements in the development of biologics. Bioanalysis. 2011;3(11):1287-95.

6. Thway TM et al. Impact of anti-drug antibodies in preclinical pharmacokinetic assessment. AAPS J. 2013;15(3):856-63.

7. Singh AP et al. Quantitative prediction of human pharmacokinetics for mAbs exhibiting target-mediated disposition. AAPS J. 2014.

8. Vugmeyster $\mathrm{Y}$ et al. Agonistic TAM-163 antibody targeting tyrosine kinase receptor-B: applying mechanistic modeling to enable preclinical to clinical translation and guide clinical trial design. MAbs. 2013;5(3):373-83.

9. Dong JQ et al. Quantitative prediction of human pharmacokinetics for monoclonal antibodies: retrospective analysis of monkey as a single species for first-in-human prediction. Clin Pharmacokinet. 2011;50(2):131-42.

10. Thway TM et al. Experimental and statistical approaches in method cross-validation to support pharmacokinetic decisions. J Pharm Biomed Anal. 2009;49(3):613-8.

11. Lee J, Ma H. Specificity and selectivity evaluations of ligand binding assay of protein therapeutics against concomitant drugs and related endogenous proteins. AAPS J. 2007;9(2):E164-70.

12. Beighton P. Sclerosteosis. J Med Genet. 1988;25:200-3.

13. Thway TM et al. In silico evaluation of the potential impact of bioanalytical bias difference between two therapeutic protein formulations for pharmacokinetic assessment in a biocomparability study. AAPS. 2015. doi:10.1208/s12248-015-9741-9.

14. Magana I et al. Validation of a micro-fluidic platform to measure total therapeutic antibodies in support of a regulated preclinical study and incurred sample reanalysis performance. Bioanalysis. 2014;6(19):2623-33.

15. Waage $\mathrm{P}$, Guldberg $\mathrm{M}$. Studies concerning affinity. Forhandlinger: Videnskabs-Selskabet i Christinia (Norwegian Academy of Science and Letters). 1864: 35.

16. Fischer SK et al. The assay design used for measurement of therapeutic antibody concentrations can affect pharmacokinetic parameters: case studies. MAbs. 2012;4(5):623-31.

17. Lobo ED, Hansen RJ, Balthasar JP. Antibody pharmacokinetics and pharmacodynamics. J Pharm Sci. 2004;93(11):2645-68. 